\title{
The Perceived Risks of Adopting a Responsible Gambling Behavior: Development and Validation of a Reliable Measurement Index (An Abstract)
}

\author{
Anne-Claire Pin, Karine Picot-Coupey, and Olivier Droulers
}

\begin{abstract}
This research aims at developing a measurement index of the perceived risks of adopting a responsible gambling behavior. It builds on existing gambling studies, the concept of perceived risk both in consumer psychology, and in health research as well as Rothman and colleagues' works on health-framed messages and contributes to address a specific research gap: the absence of a measurement instrument of the perceived risks of adopting a responsible gambling behavior. Following Churchill's, Rossiter's, and Jarvis and colleagues' recommendations regarding the development of measurement instruments, we conducted a quantitative study ( $n=22$ gamblers) in order to thoroughly conceptualize the concept of perceived risks of adopting a responsible gambling behavior. Three quantitative studies ( $n=605$ gamblers) allowed assessing the stability, the predictive, and discriminant validity of the measure. Results show that the perceived risks of adopting a responsible gambling behavior index are a valid and reliable measure formed of five dimensions: perceived risk of (1) living less excitement, (2) having less socialization, (3) earning less money, (4) winning less money, and, finally, (5) having less distraction. Results suggest that gamblers perceive responsible gambling as a risky alternative to gambling because they would not be able to satisfy their gambling motivations.
\end{abstract}

\footnotetext{
A.-C. Pin $(\bowtie)$

Université de Bretagne Sud, IREA EA 4251, Lorien, France

e-mail: anneclaire.pin@gmail.com

K. Picot-Coupey $\bullet$ O. Droulers

Université de Rennes 1, Rennes, France

e-mail: Karine.picot@univ-rennes1.fr; olivier.droulers@univ-rennes1.fr 